\title{
Clasificación de reactivos químicos en los laboratorios de la Universidad Nacional
}

\author{
Classification of chemical reagents in the \\ laboratories of National University
}

José Carlos Mora Barrantes'

Gilberto Piedra Marín

David Benavides Ramírez ${ }^{3}$

Clemens Ruepert Ruepert ${ }^{4}$

Fecha de recepción: 21 de octubre del 2011

Fecha de aprobación: 7 de abril del 2012

Mora, J; Piedra, G; Benavides, D; Ruepert, C. Clasificación de reactivos químicos en los laboratorios de la Universidad Nacional. Tecnología en Marcha. Vol. 25, N 3. Julio-Setiembre 2012. Pág 50-57.

I. Químico industrial. Escuela de Química, Universidad Nacional, Costa Rica. Teléfono: 2277-3403 Correo electrónico: jmor@una.ac.cr

2. Químico. Escuela de Química, Universidad Nacional, Costa Rica. Teléfono: 2277-3579 Correo electrónico: gpiedra@una.ac.cr

3. Químico industrial. Programa Campus Sostenible, Universidad Nacional, Costa Rica. Teléfono: 2277-3139 Correo electrónico: dbenavid@una.ac.cr

4. Químico industrial. Instituto Regional de Estudios Toxicológicos, Universidad Nacional, Costa Rica. Teléfono: 2277-350I Correo electrónico cruepert@una.ac.cr 


\section{Resumen}

Durante el periodo 2008-2010 se realizaron inventarios de los reactivos químicos utilizados y almacenados en los laboratorios de los campus Omar Dengo y Benjamín Núñez de la Universidad Nacional.

Se le solicitó a cada coordinador de laboratorio completar un formulario que incluía el nombre, la cantidad y el número CAS de los reactivos químicos almacenados y utilizados en cada laboratorio. Con estos datos, se clasificaron los reactivos de acuerdo con su categoría de peligro, utilizando el Código IMDG de la Organización de las Naciones Unidas $(\mathrm{ONU})$.

La clasificación de los reactivos químicos permitió el desarrollo de sus patrones de distribución en las diferentes unidades, institutos y centros de investigación de la Universidad Nacional. Además, se identificaron las clases de reactivos de mayor y menor uso en los laboratorios de la institución. El adecuado manejo de los reactivos químicos, con su correspondiente clasificación basada en la categoría de riesgo, es la base principal para la implementación de un ambiente seguro de trabajo en los laboratorios.

La clasificación de los reactivos químicos permite minimizar los costos administrativos, económicos, legales, de seguridad y técnicos asociados con la atención de emergencias químicas; permitiendo además el desarrollo y aplicación de prácticas de trabajo preventivas por parte de funcionarios y estudiantes durante la manipulación de estas sustancias.

\section{Palabras clave}

Reactivos químicos, sistema de clasificación química, manipulación de reactivos, salud, ambiente.

\begin{abstract}
During 2008-20I0 inventories-of chemical reagents used and stored in teaching and research laboratories of Omar Dengo and Benjamín Núñez campuses of National University were generated. E

ach laboratory coordinator was asked to fill out a form that included name, quantity and CAS number of every chemical reagent stored and utilized in the laboratories. Chemical reagents were then classified according to the risk categories described by the United Nations IMDG Code. Such a classification process allowed the development of distribution patterns of the chemical reagents within the schools, institutes and research centers of Universidad Nacional.

In addition, they were identified the chemicals hazardous classes of the reagents of larger and lesser utilization within the university laboratories. An adequate chemical reagent management, along with a classification system based upon risk categories, is necessary in order to establish a safe working environment in university laboratories.
\end{abstract}

Classification of chemical reagents permits reduction of the administrative, economical, legal, safety, and technical expenses associated with chemical emergencies management; furthermore, it allows the development and application of preventive work practices by students and university personnel during chemical reagent manipulation.

\section{Key words}

Chemical reagents, hazardous materials, identification system, chemical manipulation, environment, health. 


\section{Introducción}

Los laboratorios de investigación, enseñanza, extensión y vinculación externa de las universidades, tanto públicas como privadas, generan una gran variedad de residuos químicos peligrosos. Los volúmenes y la frecuencia con que se producen tales residuos varían en función de las actividades que se realizan en cada laboratorio. Así por ejemplo, en los laboratorios de enseñanza, las cantidades de residuos químicos pueden variar de un semestre a otro o de un año a otro, en función de los reactivos utilizados (Cortinas de Nava, 2005).

A diferencia de las actividades de la industria de la transformación, que generan volúmenes elevados y constantes de sustancias peligrosas, en los laboratorios universitarios se utilizan pequeñas cantidades, y de forma esporádica, de una gran variedad de reactivos químicos, lo que resulta en menor cantidad de residuos pero de muy diversa índole. La diversidad en los volúmenes y las características físico-químicas de los residuos requiere de una gestión integral para lograr una adecuada disposición final de estos (Cortinas de Nava, 2005).

En Costa Rica, la legislación aplicable a los vertidos industriales no se puede aplicar a los residuos provenientes de los laboratorios universitarios. Esta situación no es única para este país; la Agencia de Protección Ambiental de Estados Unidos (EPA) reconoció que la legislación desarrollada para regular los vertidos de residuos peligrosos no había sido diseñada para ser aplicada a los desechos químicos provenientes de universidades. Ante ello, la EPA se dio a la tarea de desarrollar proyectos que buscaran alternativas para reducir, reutilizar y reciclar los residuos peligrosos generados en los laboratorios universitarios, así como para darles un tratamiento o disposición final ecológicamente racional (EPA, 2002, citado por Cortinas de Nava, 2005).

Uno de los pasos iniciales de estos planes de manejo ambiental de los laboratorios consiste en que cada universidad realice la evaluación de su desempeño ambiental inicial. Esto implica identificar los residuos peligrosos y medir tanto el volumen generado como los volúmenes reutilizados o reciclados (EPA, 2002, citado por Cortinas de Nava, 2005).

Se conocen al menos cinco sistemas para clasificar e identificar la peligrosidad de las sustancias químicas utilizadas en los laboratorios de investigación y docencia y en los laboratorios de control de calidad y de ensayo, así como en la industria química de gran escala, entre otros. Tales sistemas categorizan los productos químicos de acuerdo con criterios de compatibilidad físico-química, principalmente. A continuación, se enumeran dichos sistemas (PNUMA, OMS, SAICM, 2007; National Institute for Occupational Safety and Health, 2004).

I. Agencia de Protección Ambiental de Estados Unidos (EPA, Environmental Protection Agency).

La EPA agrupa los productos o desechos químicos en cuatro categorías, como se muestra en el cuadro I (PNUMA, OMS, SAICM, 2007).

\section{Cuadro I. Sistema de clasificación de productos químicos de la EPA}

\begin{tabular}{|c|c|}
\hline Categoría & Descripción \\
\hline 1 & Sustancias inflamables \\
\hline 2 & Sustancias tóxicas \\
\hline 3 & Sustancias corrosivas \\
\hline 4 & Sustancias reactivas \\
\hline
\end{tabular}

Cuadro 2. Sistema de clasificación de productos químicos del OSHA

\begin{tabular}{|c|c|c|c|}
\hline Grupo I. Peligro físico & \multicolumn{3}{|c|}{ Grupo 2. Peligro para la salud } \\
\hline Líquidos combustibles & Gases inflamables & Cancerígenos & Neurotoxinas \\
\hline Gases comprimidos & Líquidos inflamables & Corrosivos & Toxinas de la sangre \\
\hline Explosivos & Sólidos inflamables & Altamente tóxicos & Toxinas pulmonares \\
\hline Peróxidos orgánicos & Aerosoles & Irritantes & Toxinas reproductivas \\
\hline Reactivos pirofóricos & & Tóxicos & Dañinos para la piel \\
\hline Sustancias reactivas & & Hepatotoxinas & Reactivos dañinos para los ojos \\
\hline Reactivos hidrofóricos & & Neprotoxinas & \\
\hline
\end{tabular}


Cuadro 3. Sistema de clasificación de productos químicos de la CE

\begin{tabular}{|c|c|c|c|}
\hline Categoría & Descripción & Categoría & Descripción \\
\hline 1 & Explosivos & 6 & Tóxicos \\
\hline 2 & Comburentes & 7 & Nocivos \\
\hline 3 & Extremadamente inflamables & 8 & Corrosivos \\
\hline 4 & Inflamables & 9 & Irritantes \\
\hline 5 & Muy tóxicos & 10 & Peligrosos para el ambiente \\
\hline
\end{tabular}

2. Departamento de Seguridad y Salud Ocupacional de Estados Unidos (OSHA, Occupational Safety \& Health Administration).

El OSHA definió 25 categorías de reactivos químicos peligrosos y las clasificó en dos grandes grupos: peligro físico y peligro para la salud, como se muestra en el cuadro 2 (National Institute for Occupational Safety and Health, 2004).

3. Comunidad Europea (CE).

La Comunidad Europea (CE) clasifica los reactivos químicos de acuerdo con sus propiedades

Cuadro 4. Clasificación de reactivos químicos según el Código IMDG

\begin{tabular}{|c|c|c|c|}
\hline Clase & División & Simbología & Simbología \\
\hline & $\begin{array}{l}\text { I. I: Riesgo de explosión en masa } \\
\text { ।.2: Riesgo de proyección } \\
\text { I.3: Riesgo de incendio }\end{array}$ & & $\begin{array}{c}\text { Trinitrotolueno } \\
\text { Cartuchos para armas } \\
\text { Bengalas }\end{array}$ \\
\hline Clase I. Explosivos & $\begin{array}{l}\text { 1.4: Bajo riesgo de explosión en } \\
\text { masa } \\
\text { I.5: Riesgo de explosión en masa, } \\
\text { pero son altamente insensibles } \\
\text { I.6: Contienen reactivos } \\
\text { detonantes sin riesgo de } \\
\text { explosión en masa }\end{array}$ & & $\begin{array}{l}\text { Mechas detonantes } \\
\text { Explosivos para } \\
\text { voladuras Bengalas }\end{array}$ \\
\hline Clase 2. Gases & $\begin{array}{c}\text { 2.1: Inflamables } \\
\text { 2.2: No inflamables } \\
\text { 2.3:Tóxicos }\end{array}$ & & $\begin{array}{l}\text { Propano } \\
\text { Argón } \\
\text { Amoníaco }\end{array}$ \\
\hline $\begin{array}{l}\text { Clase 3. Líquidos } \\
\text { inflamables }\end{array}$ & & & Benceno \\
\hline $\begin{array}{l}\text { Clase 4. Sólidos } \\
\text { inflamables }\end{array}$ & $\begin{array}{c}\text { 4. I: Sólidos inflamables } \\
\text { 4.2: Sólidos de combustión } \\
\quad \text { espontánea } \\
\text { 4.3: Sustancias reactivas al agua }\end{array}$ & & $\begin{array}{l}\text { Fósforo } \\
\text { Sulfuro de potasio } \\
\text { Carburo de calcio }\end{array}$ \\
\hline $\begin{array}{l}\text { Clase } 5 . \text { Oxidantes } \\
\text { y peróxidos } \\
\text { orgánicos }\end{array}$ & $\begin{array}{c}\text { 5.1: Oxidantes } \\
\text { 5.2: Peróxidos orgánicos }\end{array}$ & & $\begin{array}{c}\text { Permanganato de } \\
\text { potasio } \\
\text { Peróxido de benzoílo }\end{array}$ \\
\hline $\begin{array}{c}\text { Clase 6. Venenosos } \\
\text { e infecciosos }\end{array}$ & $\begin{array}{l}\text { 6. I: Sustancias venenosas } \\
\text { 6.2 Sustancias infecciosas }\end{array}$ & & $\begin{array}{l}\text { Cianuros } \\
\text { Ántrax }\end{array}$ \\
\hline $\begin{array}{c}\text { Clase 7. Radiactivos } \\
\text { Clase 8. Corrosivos } \\
\text { Clase } 9 . \\
\text { Misceláneos }\end{array}$ & & & $\begin{array}{c}\text { Uranio } \\
\text { Acido sulfúrico } \\
\text { Reactivo modificado } \\
\text { genéticamente }\end{array}$ \\
\hline
\end{tabular}


Cuadro 5. Número de laboratorios incluidos en el estudio

\begin{tabular}{|c|c|}
\hline Unidad académica / Centro / Instituto & Cantidad de laboratorios \\
\hline Escuela de Química & 16 \\
\hline Escuela de Medicina Veterinaria & 16 \\
\hline Escuela de Ciencias Biológicas & 9 \\
\hline Escuela de Ciencias Agrarias & 9 \\
\hline Escuela de Ciencias Ambientales & 1 \\
\hline CINAT & 3 \\
\hline IRET & 2 \\
\hline INISEFOR & 2 \\
\hline OVSICORI & 1 \\
\hline TOTAL & 58 \\
\hline
\end{tabular}

' Se incluyen los de investigación, docencia, extensión y vinculación externa.

físicoquímicas y toxicológicas y los efectos específicos sobre la salud o el ambiente. Las categorías de sustancias y preparados peligrosos según la CE se enumeran en el cuadro 3 (Reinhart, 2009, Pipitone, 1991, National Academic Press, 1995).

4. Código IMDG (International Maritime Dangerous Goods Code) de la Organización de las Naciones Unidas (ONU).

Este sistema divide los productos, materiales o desechos peligrosos en nueve clases, las cuales están segregadas en diferentes divisiones según sus características físicas y químicas, tal y como se ilustra en el cuadro 4 (Programa de las Naciones Unidas para el Medio Ambiente, 2002).

\section{Sistema Globalmente Armonizado de la ONU}

El Sistema Globalmente Armonizado (GHS, Globally Harmonized System) es el más reciente y clasifica los productos químicos de acuerdo con tres criterios: Riesgos físicos, Riesgos para la salud y Riesgos para el medio ambiente. Cada uno de estos grupos se subdivide en diferentes categorías (Universidad de Brasilia, 2008).

En años recientes, la Universidad Nacional (UNA) se ha dado a la tarea de implementar un plan de manejo de los residuos tóxicos que se generan en sus laboratorios de análisis, enseñanza, extensión y vinculación externa. Como parte de este plan, se hizo una clasificación de los reactivos químicos utilizados en los laboratorios de la UNA, cuyos resultados se reportan en el presente trabajo.

\section{Metodología}

La clasificación de las sustancias químicas de la UNA se enfocó en los reactivos utilizados en los laboratorios de docencia, investigación, extensión y vinculación externa de los campus Omar Dengo y Benjamín Núñez. La población inicial del estudio se estableció en el 2008, utilizando información facilitada por la Vicerrectoría Académica y la Dirección de Investigación; la población de estudio se actualizó en 2010 con información procedente de las unidades académicas y decanaturas involucradas. En el cuadro 5 se detalla el número de laboratorios incluidos en el estudio.

Para realizar el inventario y la clasificación de las sustancias, se le solicitó a los coordinadores de los laboratorios llenar un formulario que incluía el nombre, la cantidad y el número CAS (Chemical Abstract Service) de todos los reactivos químicos almacenados y utilizados en ellos.

Luego se recolectaron los formularios y se revisó la información obtenida con los funcionarios de cada laboratorio; seguidamente se procedió a clasificar los reactivos químicos según el Código IMDG de la ONU. La principal fuente de información utilizada para asignar las categorías de peligro de las sustancias incluidas en el estudio fue la hoja de seguridad (Material Safety Data Sheet, MSDS) de cada reactivo. Para obtener las hojas de seguridad se visitaron los sitios web oficiales de la EPA, el OSHA, la CE y la ONU, se visitó la Proveeduría Institucional de la UNA y se solicitó tal información a los proveedores nacionales de reactivos químicos. 
A partir de la clasificación, se generó una base de datos y patrones de distribución de los reactivos químicos almacenados y consumidos durante el periodo 2008-2010.

\section{Resultados y discusión}

El $83 \%$ de los 58 laboratorios incluidos en el estudio completó los formularios con la información solicitada. Se determinó que en los laboratorios y bodegas se almacenaba un total de 10.218 kilogramos ( $\mathrm{kg}$ ) de reactivos químicos en forma sólida y 10.773 $L$ de reactivos líquidos, distribuidos en las diferentes unidades académicas.

La distribución porcentual del total de los reactivos sólidos y líquidos en las diferentes unidades se muestra en la figura I. Los institutos IRET, INISEFOR y CINAT aportaron cantidades inferiores al $1 \%$ del total. Por el contrario, en la Escuela de Química se localizó la mayor cantidad de reactivos químicos, aportando el $79 \%$ de los reactivos sólidos y el 71\% de los líquidos.

La mayor cantidad de reactivos químicos de la Escuela de Química obedece principalmente a que esta cuenta con 16 laboratorios, 12 de los cuales son de investigación, dos de docencia y dos de vinculación externa. Por el contrario, los institutos
IRET, CINAT, INISEFOR y OVSICORI y la escuela de Ciencias Ambientales cuentan con tres laboratorios como máximo.

Las escuelas de Ciencias Biológicas, Ciencias Agrarias y Medicina Veterinaria tienen más de seis laboratorios cada una (por ej., Medicina Veterinaria tiene 16); no obstante, sus procesos son principalmente microbiológicos, por lo que se utilizan cantidades menores de reactivos químicos en comparación con la Escuela de Química. Además, esta última escuela ofrece cursos (teoría y laboratorio) para las carreras de Química Industrial, Topografía, Ciencias Geográficas, Ciencias Biológicas, Veterinaria, Ciencias Agrarias e Ingeniería Forestal, entre ellos química general, química orgánica, química analítica, química inorgánica y bioquímica.

Como se mencionó anteriormente, los reactivos químicos se clasificaron de acuerdo con las categorías establecidas por el Código IMDG de la ONU. La distribución porcentual de cada una de las categorías, tanto para los reactivos en estado sólido como líquido, se muestra en la figura 2. La mayor cantidad (63\%) de reactivos líquidos corresponde a la clase 3 (productos químicos inflamables), mientras que en los sólidos el 33\% corresponde a la subclase 6.1 (productos químicos tóxicos). Las sustancias radiactivas (clase 7 ) son las que aportan menor porcentaje (inferior a 0,5\%) al total.

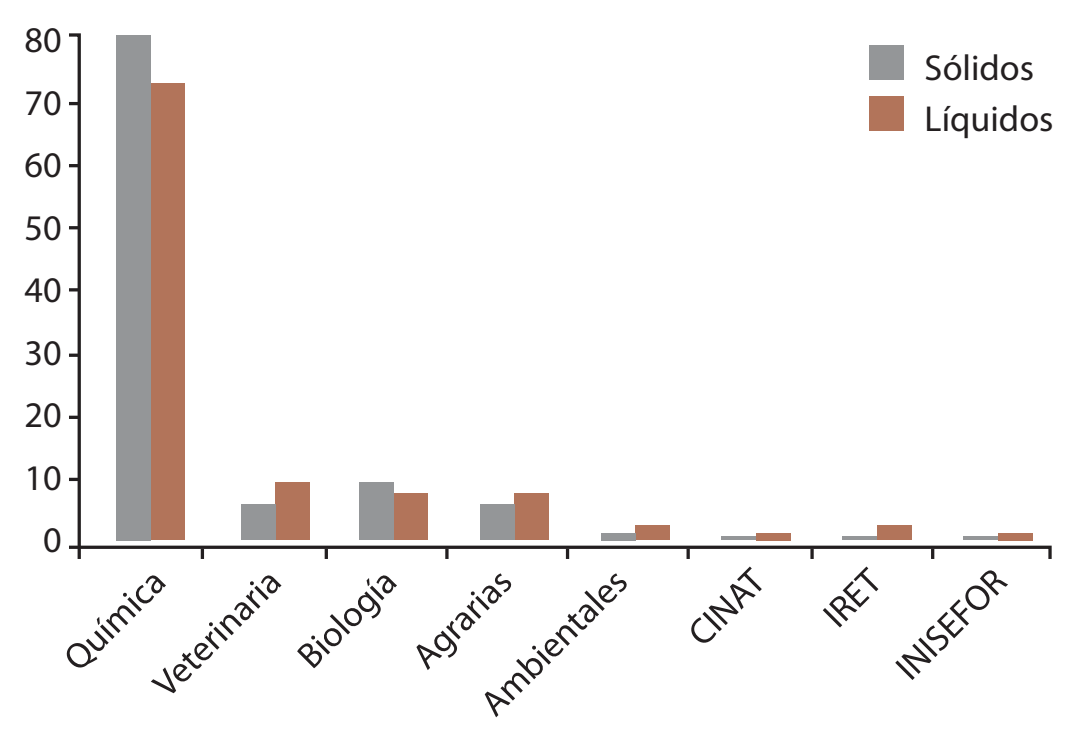

Figura I. Distribución porcentual de la cantidad de reactivos químicos almacenados en la UNA. 


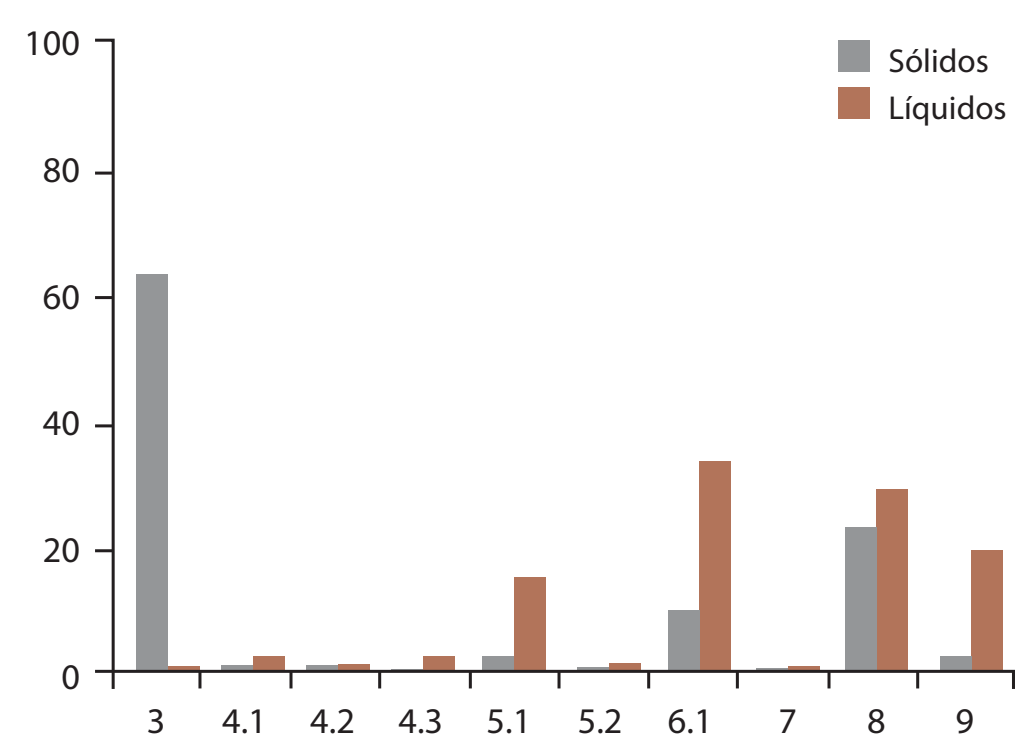

Figura 2. Distribución porcentual de reactivos químicos almacenados en la UNA, clasificados según el Código IMDG.

El alto porcentaje de los reactivos inflamables se debe al gran volumen de compuestos orgánicos que se utilizan en los diferentes laboratorios, como los disolventes orgánicos hexano, metanol, etanol, acetonitrilo y éter etílico, los cuales se usan principalmente en la Escuela de Química (LAPRONEB, LAFIT, Bioquímica, núcleo instrumental, etc.). Los disolventes anotados representan el $46 \%$ del total de los reactivos inflamables incluidos en el inventario y se usan comúnmente en análisis químicos, síntesis orgánicas y técnicas cromatográficas como HPLC.

Las escuelas de Ciencias Biológicas y Medicina Veterinaria tienen una alta demanda de etanol para sus ensayos microbiológicos, lavado de cristalería y conservación de muestras.

Otro grupo de reactivos líquidos que muestra un alto porcentaje de consumo es el de las sustancias corrosivas (23\%), debido al uso de ácidos y bases utilizados en el $80 \%$ de los laboratorios evaluados. Los ácidos clorhídrico, sulfúrico, nítrico, perclórico y acético se utilizan principalmente para acidificar mezclas y digerir muestras de suelos y sedimentos, así como en prácticas de docencia de química general y química analítica. En particular, el ácido sulfúrico presenta un alto consumo en los análisis de demanda química de oxígeno (DQO) y en la preparación de mezcla sulfocrómica para limpieza de cristalería, principalmente en la Escuela de Química.
Otros laboratorios, como los del IRET, generalmente limpian la cristalería con agua y jabón, en tanto la que se emplea en el análisis de trazas se limpia con acetona y etanol.

Con respecto a los reactivos en estado sólido, el mayor porcentaje (figura 2) corresponde a la clase 6.I, sales tóxicas, las cuales se utilizan en investigación y docencia, por ej., sales de mercurio, arsénico, cadmio y amonio. Las sales de mercurio provienen de los sulfatos utilizados en el análisis de DQO. Otros compuestos tóxicos corresponden al bromuro de etidio, el fenol y la formalina.

El bromuro de etidio se utiliza en tinción de geles y electroforesis y el fenol en tinciones y extracción de ADN, ambos en las escuelas de Medicina Veterinaria, Ciencias Agrarias y Ciencias Biológicas. La formalina o formaldehído se utiliza normalmente para la conservación de cadáveres de animales en la Escuela de Medicina Veterinaria y de plantas e insectos en la Escuela de Ciencias Biológicas.

Los compuestos sólidos corrosivos almacenados representan el $28 \%$ del total y corresponden principalmente a los hidróxidos de sodio, calcio, magnesio, aluminio y potasio, los cuales se utilizan principalmente en las prácticas de docencia, así como en titulaciones ácido-base y en los análisis de dureza en laboratorios de investigación. Existe un alto porcentaje de la clase 9, compuestos varios, los cuales no tienen una clasificación específica, o no se 
encontró la clasificación respectiva en las etiquetas y hojas de seguridad química de estos o no fue posible encontrar una clasificación en las fuentes consultadas.

La adecuada clasificación de los reactivos químicos manipulados por investigadores, técnicos, estudiantes, etc., favorece un mayor conocimiento de las propiedades físico-químicas de las sustancias. Lo anterior permite, en el mejor de los escenarios, reducir los costos administrativos, económicos, legales, de seguridad y técnicos, entre otros, relacionados con la atención de emergencias químicas (Furr, 2000, Ewing, 1990, DiBerardinis et al., 200 I).

La clasificación de los reactivos químicos en los laboratorios de los campus Omar Dengo y Benjamín Núñez de la UNA permite implementar procesos y procedimientos para la prevención del riesgo, como: desarrollo de temas de capacitación para el uso de reactivos específicos (inflamables, corrosivos, etc.), compra y mantenimiento preventivo de equipos y materiales de seguridad, aplicación de sistemas de segregación de reactivos en los sitios de trabajo, inclusión de modificaciones infraestructurales en los lugares de trabajo y uso de equipo de protección personal, entre otros. Todo ello mejora la protección de la salud de los trabajadores, estudiantes y visitantes, así como la propiedad estructural y el ambiente.

\section{Conclusiones}

El presente trabajo permitió inventariar y clasificar los reactivos utilizados en los laboratorios de los dos principales campus de la UNA. El inventario de los reactivos permitió identificar patrones de distribución, ubicación y manipulación de estos en las diferentes unidades académicas, centros e institutos, identificándose de esta manera los sitios que más manipulan productos químicos y los reactivos que se utilizan en mayor cantidad.

Durante el periodo 2008-2010 se inventariaron $10.218 \mathrm{~kg}$ y $10.773 \mathrm{~L}$ de reactivos sólidos y líquidos, respectivamente; las mayores cantidades se generaron en la Escuela de Química.

La mayor cantidad de reactivos líquidos y sólidos incluidos en el inventario corresponde a las clases 3 -productos químicos inflamables- y 6.1 -productos químicos tóxicos-, respectivamente.

El manejo adecuado de los reactivos químicos y la correspondiente clasificación físico-química basada en el riesgo y peligro de estas sustancias constituye la base para implementar un ambiente seguro de trabajo en los laboratorios universitarios.

\section{Agradecimientos}

Al personal académico y administrativo de la Universidad Nacional.

\section{Bibliografía}

Cortinas de Nava, C. (2005). Ideas sobre planes de manejo de residuos peligrosos de laboratorios universitarios: responsabilidad social de las universidades. Revista de la Universidad Cristóbal Colón, Número 20, 85-97.

Decreto 26805-S. (1998). La Gaceta 63. Reglamento sobre registro y control de productos peligrosos. San José: La Gaceta.

Decreto 13466-TSSN. (1982). La Gaceta 24. Reglamento general de seguridad e higiene de trabajo. San José: La Gaceta.

DiBerardinis, J.; Baum, J.; First, M.; Gatwood, G., \& Seth, A. (200I). Guidelines for laboratory design: Health and safety considerations. 3 ed. New York: John Wiley \& Sons, Inc.

Ewing, G. (1990). Safety in the analytical laboratory. J. Chem. (ed.), 67 (6): Al58-AI 60.

Furr, A. (2000). CRC Handbook of laboratory safety. 5 ed. Florida: CRC Press Inc.

Grupo Coordinador Nacional. (2008). Perfil nacional de la gestión racional de sustancias químicas. I ed. San José: EUNA.

National Academies Press. (1995). Prudent practices for handling hazardous chemicals from laboratories. Washington, D.C.

National Institute for Occupational Safety and Health. (2004). Pocket guide to chemical hazards. Ohio, US: NIOSH Publications.

Pipitone, D.A. (199|). Safe storage of laboratory chemicals. 2 ed. New York: John Wiley \& Sons, Inc.

PNUMA, OMS, SAICM. (2007). Enfoque estratégico para la gestión de productos químicos a nivel internacional. Ginebra.

Programa de las Naciones Unidas para el Medio Ambiente. (2002). Convenio de Basilea sobre el control de los movimientos transfronterizos de los desechos peligrosos y su eliminación. Sexta Reunión. Ginebra, pp. 36-49.

Reinhart, P. (2009). An Update of Prudent Practices in the Laboratory: Handling and Disposal of Chemicals. Yale Environmental Health \& Safety. US: National Academies Press.

Universidad de Brasilia. (2008). International Symposium on Residue Management in Universities. Brasil. 